\title{
Mi hijo y sus hermanos: la experiencia de primera maternidad al interior de familias simultáneas chilenas
}

My child and his siblings: Women's experience
of first maternity in Chilean stepfamilies

Recibido: noviembre 12 de 2009 ～Revisado: mayo 12 de 2010 | Aceptado: octubre 1 de 2010

\author{
J. CARola Pérez Ewert*** \\ ANDREA JARAMILLO TORRÉNS ${ }^{* * *}$ \\ Pontificia Universidad Católica de Chile
}

\section{RES UMEN}

Se presenta un estudio cualitativo que da cuenta de la percepción de mujeres chilenas de 29 a 39 años de edad, sobre la experiencia de tener su primer hijo al interior de una familia simultánea. Se entrevistó a 16 mujeres, cuyas parejas tenían hijos de relaciones previas y mantenían un contacto activo con ellos. Los resultados indican la existencia de un proceso para "llegar a ser madres", cuyo curso se ve afectado por la presencia de los hijastros. Además, los hallazgos muestran que se presentan motivaciones y temores específicos asociados al contexto particular de familia, variando la experiencia de llegar a ser madres acorde con la cualidad percibida en las relaciones entre ellas y sus hijastros.

Palabras clave autores

Familias simultáneas, primera maternidad, relaciones madrastras/hijastros.

Palabras clave descriptores

Psicología del desarrollo, afectividad, desarrollo afectivo, relaciones interpersonales, comportamiento de grupo.

\section{A B S T R A C T}

This qualitative study shows the perception of a group of Chilean women aged between 29 and 39 years regarding their experience of having their first child when they are members of a simple stepfamily. Sixteen women, whose partners maintain active contact with their child(ren) born in previous relationships were interviewed. Results confirm the existence of a process of "becoming a mother" and its course depends on stepchildren's presence. In addition our more relevant findings show that the specific topics of fears and motivations of having a child depend on the particular stepfamily context, and women's experiences is that this process vary according to the perceived quality of stepmother/stepchildren relationships.

Key words authors

Stepfamilies, Fist Maternity, Stepmother/Stepchildren Relationships.

Key words plus

Developmental Psychology, Emotions, Emotional Development, Interpersonal Relations, Group Behaviour. 
La familia simultánea es definida como aquella unidad familiar en la cual - al menos uno de los miembros de la pareja conyugal - tiene uno o más hijos de una relación previa (ya disuelta), sobre quien ejerce una relación de responsabilidad (Visher \& Visher, 1988).

Bumpass, Raley y Sweet (1995), señalan que un $40 \%$ de las mujeres y un $30 \%$ de los niños en Estados Unidos de América, forman parte de una familia simultánea durante su vida. En Chile, no se dispone de datos censales sobre este fenómeno, no obstante, los estudios disponibles muestran una incidencia de este tipo de familia que oscila entre el 9,2 y 14\% (Muñoz \& Reyes, 1997).

El estudio de la incorporación de nuevos miembros por nacimiento, en estas familias, ha tenido dos focos prioritarios. Desde un punto de vista demográfico, se ha puesto atención a la tasa de fertilidad y a la influencia de la presencia de hijastros $^{1}$ en las intenciones y conducta para tener hijos. Desde una perspectiva psicológico/relacional, se han analizado las consecuencias que tienen estos nacimientos, sobre el bienestar individual de los miembros del sistema familiar y sobre la calidad de la relación conyugal.

No obstante, existe escasa información sobre cómo la presencia de los hijastros afecta las intenciones y la conducta reproductiva, cuál es el desarrollo de estas intenciones y conducta a lo largo del tiempo hasta la concreción de la maternidad, y si existe alguna relación entre este desarrollo y sus consecuencias relacionales en los distintos miembros del sistema familiar. El presente estudio profundizó en la experiencia de mujeres, con miras a aportar una mejor comprensión de la conducta reproductiva y la maternidad en este particular contexto familiar, y en busca de dar cuenta de su posible impacto en la salud mental de las mujeres. Concretamente, las preguntas que guiaron el estudio fueron: ¿Distinguen las mujeres un proceso en su experiencia de primera maternidad en este tipo

1 Se asume la importancia de la distinción lingüística de género, sin embargo, dado que los resultados de la investigación realizada por las autoras no mostraron diferencias según sexo en hijos/as ni hijastros/as, se obviará dicha distinción para facilitar la lectura del texto. de familias?, ¿Cuáles son los marcadores o etapas consideradas relevantes?, ¿La presencia de hijastros tiene alguna ingerencia en la vivencia del proceso de llegar a ser madres?, y -en caso de presentarse¿cómo es esa influencia?

El estudio fue realizado en Santiago de Chile, país que se caracteriza por la coexistencia de valores familiares tradicionales, junto a aquellos más liberales que tienden a presentarse en los países desarrollados. Por ejemplo, la conducta reproductiva en Chile se ha caracterizado por una disminución en la tasa de nacimientos y por una tendencia de la mujeres a posponer la maternidad (Instituto $\mathrm{Na}$ cional de Estadísticas [INE], 2005). Estos cambios en los patrones reproductivos coexisten con valores tradicionales, de hecho, la maternidad continúa siendo un aspecto central en los proyectos vitales de las mujeres chilenas (Servicio Nacional de la Mujer [SERNAM], 2002).

\section{Motivaciones para ser madre al interior de una familia simultánea}

Investigación demográfica desarrollada en USA indica que un gran número de niños nace en los nuevos matrimonios de mujeres que ya tienen hijos nacidos en el seno de sus primeros matrimonios. Wineberg (1992) señala que cerca del $50 \%$ de las mujeres que se casa por segunda vez, da a luz otros hijos, y que estos nacimientos acontecen temprano en el curso de la relación (33 \% nace dentro de los primeros 24 meses).

Estudios han mostrado que las intenciones de tener hijos estarían presentes al conformar nuevas parejas, pero estas se verían afectadas por la presencia de hijastros. Específicamente en el caso de las mujeres, los resultados indican que los hijos de relaciones previas reducen la probabilidad de que tengan hijos con una nueva pareja (Loomis \& Landale, 1994; Wineberg, 1990). Resultados similares se presentan en el caso de los hombres, ya que sus hijos nacidos en relaciones previas reducen la probabilidad de tener "un hijo en común" con su nueva pareja (Haurin, 1994).

Stewart (2002) profundizó en el efecto que tiene la presencia de hijastros sobre las intenciones 
y conducta reproductiva en familias simultáneas, considerando el sexo de cada cónyuge y la diversidad estructural de este tipo de familias. Los resultados muestran que en familias simultáneas, donde ambos miembros de la pareja conyugal tienen hijos de relaciones previas, la presencia de los hijastros disminuye las intenciones de tener hijos, comparado con aquellas familias donde un solo miembro tiene hijos de relaciones previas (familias simultáneas simples). Además, la presencia de hijastros disminuye las intenciones de tener un hijo de manera más significativa que la presencia de hijos biológicos propios, patrón que varía según el sexo del progenitor. Para las madres, tanto los hijastros como sus hijos biológicos afectan de igual manera la intención de tener más hijos; en cambio en los padres, los hijastros reducen las intenciones de tener más hijos, pero sus hijos biológicos no lo hacen.

Estos resultados sugieren que la presencia de hijastros puede influenciar la tasa de fertilidad de la familia simultánea, al afectar las intenciones de tener hijos, pero se desconoce efectivamente cómo se produce este impacto (Stewart, 2002). De hecho, en la base de estos estudios, se encuentra un modelo cognitivo que estipula que las intenciones de conducta implican un involucramiento personal para actuar, el cual se traduciría en la conducta reproductiva. Cuando las condiciones y el momento sean adecuadas, las intenciones serían transformadas en conductas instrumentales que se traducen en el logro de la concepción (Miller, 1992). No obstante, cuál es el minuto y las condiciones adecuadas en este tipo de familias, y cómo estas intenciones pueden verse afectadas por la presencia de hijastros, no ha sido estudiado, constituyendo uno de los aportes del presente artículo.

Thompson y Allen Li (2002) plantean que los nacimientos en las familias simultáneas adquieren diferentes significados para sus miembros. Por ejemplo, el nacimiento del "primer hijo en común" puede ser un signo de compromiso de la nueva pareja conyugal, y momento en que alguno de ellos puede adquirir un nuevo rol (ser madre/padre por primera vez). Adicionalmente, indican que el nacimiento del "segundo hijo en común" permite conformar un sistema filial completo, en donde dos hermanos comparten a ambos progenitores. Estas representaciones asociadas al nacimiento "del primer hijo en común" son sopesadas con los costos de la crianza (económicos y dedicación de tiempo) al momento de decidir tener hijos.

$\mathrm{Al}$ estudiar las intenciones de tener hijos y conducta reproductiva de diferentes constelaciones de familias, los mismos autores Thompson y Allen Li (2002), reportan que no se encuentran diferencias en las intenciones de tener hijos y en la probabilidad de nuevos nacimientos al comparar familias con un solo hijo. Por ejemplo, familias simultáneas simples con un hijastro vs. familias nucleares con un hijo. En cambio, al comparar diferentes familias simultáneas que tienen dos o más hijos, se presentan intenciones más marcadas y mayor probabilidad de nacimientos, en aquellas parejas que no tienen un "hijo en común", particularmente si es la mujer quien no ha tenido hijos biológicos. Sobre la base de estos resultados, los autores concluyen que existe una valoración especial y singular asociada al nacimiento del "primer hijo en común" en las familias simultáneas (Thompson \& Allen Li, 2002).

Estos resultados sugieren que aquellas familias simultáneas donde las mujeres -que al momento de establecer la relación de pareja- no han sido madres biológicas, constituyen un punto de partida para la compresión de este fenómeno. Asumiendo la existencia de una valoración particular del "primer hijo en común", se espera que aparezcan las intenciones de tener hijos, permitiendo conocer cómo evolucionan estas intenciones hasta su traducción en la conducta reproductiva, e identificar las condiciones y el momento adecuado para su concreción en el contexto de familia simultánea, y -finalmente- determinar cómo la presencia de hijastros impacta este proceso.

\section{Embarazo y maternidad en familias simultáneas}

Tradicionalmente se ha considerado que la maternidad es la fuente de identidad más poderosa de las mujeres (Rogers \& White, 1998). Sin embargo, si 
bien tener un hijo es considerado un evento positivo, la investigación indica que este constituye un evento potencialmente estresante para los padres y requiere de un período de ajuste. De hecho, la llegada de un hijo a un hogar se ha asociado con un descenso en la satisfacción marital (Demo \& Cox, 2000) y un incremento en la sintomatología psicológica, particularmente en las nuevas madres (Cowan \& Cowan, 1995).

Dietz-Omar (1991) comparó la experiencia de familias nucleares con familias simultáneas durante el embarazo de su "primer hijo en común". Sus resultados indican que diferentes aspectos del funcionamiento familiar se relacionan con la satisfacción de las parejas en ambos tipos de familias. Particularmente, en las familias simultáneas el contar con estrategias de afrontamiento ante las dificultades favorece la satisfacción en las parejas, en cambio, en las familias nucleares una mayor cohesión se traduce en una mayor satisfacción con la vida familiar.

La cohesión familiar es definida como la ligazón emocional que los miembros de una familia tienen entre sí (Olson, Russell \& Sprenkle, 1983). Lograr la cohesión familiar constituye uno de los tópicos evolutivos de las familias simultáneas. De hecho, Visher y Visher (1988) señalan que la tarea básica de las familias simultáneas consiste en lograr la transición satisfactoria desde sus culturas familiares previas, a la construcción de una cultura común de la nueva familia, una nueva identidad familiar. Lograrlo requiere, entre otros aspectos, formar relaciones satisfactorias entre los diversos miembros de la familia; particularmente entre aquellos miembros que no comparten lazos biológicos o de desarrollo, como lo son las relaciones de madrastra o padrastro con sus hijastros, y las relaciones entre hermanastros.

Estos resultados indican que las relaciones entre miembros no ligados biológicamente (como el caso de mujeres y sus hijastros) afectan la satisfacción marital y, en este sentido, podrían impactar la vivencia asociada al embarazo y primera maternidad en mujeres que conforman pareja con hombres con hijos nacidos en relaciones previas. En el presente estudio, la manera en que estas relacionen im- pactan la vivencia de la primera maternidad, será analizada desde una mirada cualitativa.

\section{Consecuencias de tener el "primer hijo en común"}

Crosbie-Burnett (1989) postula que el nacimiento de un hijo en una familia simultánea es un estresor, cuyo impacto en su funcionamiento - adaptación vs. desadaptación - va a depender de los recursos individuales, familiares y de redes con que esta cuenta, así como de la percepción subjetiva de sus miembros. Coherente con ello, una variedad de estudios se han concentrado en las consecuencias derivadas del nacimiento de estos hijos (por ejemplo, Ganong \& Coleman, 1988). No obstante, estos estudios presentan resultados diferentes o contradictorios sobre el impacto que tiene el nacimiento de un nuevo miembro en estas familias sobre las relaciones entre miembros no ligados biológicamente, en el grado de certeza o claridad del rol que los adultos tienen que desempeñar y en la satisfacción marital.

En uno de los primeros estudios disponibles, realizado por Duberman (1973), se indica que en familias simultáneas que tienen hijos en común las relaciones entre hijastros y padrastros son mejores. En cambio, Ganong y Coleman (1988) indican que no hay diferencias en el grado de cercanía y afecto entre familias simultáneas que poseen y no poseen un "hijo en común". En forma más reciente, Stewart (2005) concluye que el nacimiento de un nuevo hijo no mejora el nivel de involucramiento en actividades comunes entre hijastros y padrastros.

Ambert (1986), quien estudió cualitativamente el impacto del nacimiento de un "hijo en común" dentro de una familia simultánea, cuando los hijastros y este nuevo miembro viven en el mismo hogar, señalando que los padrastros se benefician con este nacimiento en el ámbito relacional. En cambio, para las madrastras dicho nacimiento genera distancia de sus hijastros, ya que ellas desean concentrarse más en la crianza del "propio hijo".

Por último, Downs (2003) encontró que la presencia de un "hijo en común", no afecta el grado 
de certeza y claridad que las madrastras y padrastros tienen de su rol. Lo que sería contradictorio con lo planteado por Fine (1995), quien plantea que actuar simultáneamente los roles parentales hacia hijos biológicos e hijastros, puede exacerbar la ambigüedad del rol.

Contradictorios son también los resultados sobre el impacto del nacimiento del "hijo en común" en la satisfacción marital. Ganong y Coleman (1988) no encontraron diferencias en el nivel de satisfacción marital entre parejas que hubiesen o no tenido este hijo. En cambio, Downs (2003) encontró que cuando había un hijo mutuo en estas familias, los adultos percibían a sus parejas como menos apoyadoras y presentaban creencias de que "otras familias" serían más atractivas que la propia, es decir, estarían menos satisfechas.

Dada la diversidad de constelaciones posibles dentro de estas familias, los diversos actores involucrados y los resultados de los estudios demográficos, el presente estudio cualitativo se focalizó en familias simultáneas simples, donde la mujer no aportaba inicialmente hijos al sistema familiar. Además, atendiendo a la tendencias observadas en las mujeres chilenas de posponer su maternidad, y mayor participación en el mundo laboral (Tironi et al., 2003), se decidió realizar este estudio desde la perspectiva de mujeres profesionales de alrededor de 35 años de edad.

$\mathrm{Al}$ revisar la experiencia de estas mujeres de llegar a ser madres, el presente artículo focaliza la atención en el devenir de las relaciones conyugales en este proceso, y da cuenta de la ingerencia de la presencia de los hijastros en la vivencia asociada a ello. Se detiene así en las relaciones madrastras/ hijastros, y en las relaciones entre medios hermanos que comienzan a desarrollarse, aportando a la comprensión de las diferencias entre los hallazgos antes mencionados.

\section{Método}

El presente estudio descriptivo-relacional fue desarrollado en Santiago de Chile, basado en la Teoría Fundada. Se focaliza en un tipo particular de familia simultánea, conformada por mujeres que no ha- biendo tenido hijos, se casaron (o formaron pareja estable) con hombres que tenían hijos de relaciones previas. A través del uso de esta metodología, se buscó dar cuenta de la manera particular en que las mujeres perciben e interpretan sus experiencias en torno a la maternidad en este contexto familiar, y crear una perspectiva teórica que dé cuenta de estos tópicos (Flick, 2002).

\section{Participantes}

Las participantes fueron seleccionadas siguiendo los procedimientos del Muestreo Teórico (Flick, 2002; Glasser \& Strauss, 1967). Adicionalmente, se usaron técnicas de muestreo en cadena o bola de nieve para identificar y contactar participantes (Taylor \& Bogdan, 1984), y muestreo por intensidad para profundizar en algunos tópicos (Flick, 2002).

Los criterios iniciales de selección de las participantes fueron (a) ser mujer profesional, treintañera, sin hijos al momento de conformar su familia simultánea, (b) casada o conviviendo con hombre separado que ejerce relación de responsabilidad con uno o más hijos menores de 18 años, a través del aporte económico y/o visita sistemáticas, (c) tener al menos un hijastro viviendo con su madre biológica, el cual visita su hogar dos o más veces al mes, concordando con la realidad de las familias separadas en Chile (Muñoz \& Reyes, 1997).

A medida que se fueron analizando las entrevistas, emergieron criterios adicionales para continuar con el procedimiento de muestreo, siendo los tres los más relevantes: (1) presentar problemas de fertilidad, (2) estar en el proceso de decidir si tener o no hijos y (3) estar tratando de embarazarse.

Las participantes de este estudio fueron $16 \mathrm{mu}$ jeres, entre 29 y 39 años. Nueve mujeres habían tenido su hijo al momento de la entrevista. De las siete mujeres que no habían tenido hijos al momento de la entrevista, dos presentaban problemas de fertilidad, tres de ellas se encontraban en el proceso de decidir si tener o no hijos, y dos se encontraban tratando de embarazarse. Ellas tenían entre uno y tres "hijastros", de diferentes edades (preescolares, escolares y adolescentes). 
TABLA 1

Características de las mujeres, hijastros e hijos (en caso de tenerlos)

\begin{tabular}{|c|c|c|c|c|}
\hline \multicolumn{5}{|c|}{ Mujeres que habían sido madres } \\
\hline \multicolumn{4}{|c|}{ Mujer } & \multirow{2}{*}{$\begin{array}{c}\text { Hijastros } \\
\text { Edad y sexo }\end{array}$} \\
\hline $\mathrm{N}^{\circ}$ & Edad $^{\mathrm{a}}$ & Duración de la relación ${ }^{\mathrm{a}}$ & Edad y sexo del hijo propio & \\
\hline 1 & 37 & 3 & 2 años, Mujer (M) & 7 años, $M$ \\
\hline 2 & 33 & 3 & 1 año, Hombre $(\mathrm{H})$ & 10 años, $M$; 6 años, $H$ \\
\hline 3 & 33 & 5 & 3 años, $M$ & 12 años, $M^{\Delta}$ \\
\hline 4 & 33 & 5 & 7 meses, $\mathrm{M}$ & 14 años, $\mathrm{H} ; 11$ años, $M$ \\
\hline 5 & 35 & 3 & 9 meses, $\mathrm{H}$ & 10 años, $H^{\Delta}$ \\
\hline 7 & 30 & 2 & 10 Meses, $\mathrm{M}^{\tau}$ & 11 años, $\mathrm{H}$; 9 años, $M$ \\
\hline 8 & 39 & 10 & 6 años, $\mathrm{H}$ & 20 años, $H$ \\
\hline 13 & 35 & 3 & 10 meses, $M$ & 12 años, $M^{\Delta}$ \\
\hline 15 & 34 & 7 & 5 años, $\mathrm{H}$ & 10 años, $M$ \\
\hline \multicolumn{5}{|c|}{ Mujeres que no habían sido madres } \\
\hline \multicolumn{4}{|c|}{ Mujer } & Hijastros \\
\hline $\mathrm{N}^{\circ}$ & Edad $^{\mathrm{a}}$ & Duración de la relación ${ }^{\mathrm{a}}$ & Etapa en el proceso de "ser madre" & Edad y sexo \\
\hline 6 & 32 & 3 & Problemas de fertilidad & 18 años, $M$; 16 años, $M ; 8$ años, $H$ \\
\hline 9 & 37 & 5 & Problemas de fertilidad & 23 años, $\mathrm{H}$; 15 años, $M$; 12 años, $M$ \\
\hline 10 & 35 & 8 & Intentando embarazarse & 12 años, $M$ \\
\hline 11 & 36 & 3 & Tomando la decisión & 14 años, $M^{\Delta}$ \\
\hline 12 & 38 & 3 & Tomando la decisión & 23 años, M (hijastra residente) \\
\hline 14 & 38 & 2 & Tomando la decisión & 17 años, $M ; 16$ años, $M ; 9$ años, $H^{\Delta}$ \\
\hline 16 & 29 & 3 & Intentando embarazarse & 9 años, $\mathrm{H}$ \\
\hline
\end{tabular}

${ }^{\Delta}$ Relaciones entre mujeres y sus hijastros distantes o conflictivas

${ }^{\tau}$ Embarazo no planificado

${ }^{a}$ Edad (en años) al momento de la entrevista

Fuente: elaboración propia

\section{Procedimientos}

Acorde con los procedimientos de rigor metodológico de los estudios cualitativos (Flick, 2004), la selección de las participantes, la recolección de los datos y el análisis de los mismos, se implementaron de manera simultánea y recursiva.
Las mujeres fueron inicialmente contactadas telefónicamente, se les explicaban los objetivos del estudio, se les clarificaba la naturaleza voluntaria de su participación y el uso confidencial de la información recolectada; adicionalmente se verificaba que cumplieran los criterios de inclusión. En dicha instancia, se acordaba una fecha de entrevista, instancia en la cual se firmaba el consentimiento 
informado. Las entrevistas fueron desarrolladas en las propias casas de las entrevistadas, su trabajo o en un café, de acuerdo con las preferencias de las entrevistadas. Las entrevistadas fueron grabadas en audio, transcritas y analizadas.

Inicialmente, se contactó a 3 mujeres que ya habían sido madres al momento de la entrevista, realizando relatos retrospectivos sobre la decisión de tener hijos, el proceso de concebir y las consecuencias relacionales en el sistema familiar del nacimiento de su hijo. Con base en el análisis de contenido de estas entrevistas, se determinó incluir 2 mujeres con problemas para concebir, dada la importancia identificada de este factor en la experiencia de maternidad. Un segundo conjunto de 4 participantes que ya tenía su "primer hijo en común" fueron contactadas, complementando la información recolectada y elaborándose hipótesis más específicas respecto del proceso de tener hijos.

Acorde a la información recolectada, un tercer grupo de mujeres fue contactado, requiriéndose que se encontraran en proceso de decidir o de tener su primer hijo. Tres mujeres fueron entrevistadas, y se acordó con ellas llevar a cabo una segunda entrevista, en un plazo de 3 a 4 meses, con el objetivo de seguir la evolución de su particular proceso de decisión o de "campaña para tener hijos" (lenguaje coloquial que se usa para denotar el período en que las parejas se encuentran tratando de tener hijos). En la fecha acordada se realizó la entrevista de seguimiento.

Por último, y a fin de saturar las categorías de análisis aún incompletas, el último grupo de mujeres que se contactó fue heterogéneo, buscando incluir tanto a mujeres que no tenían todavía su primer hijo, como aquellas que ya habían sido madres, con diferentes grados de satisfacción con sus hijastros (buenas y malas relaciones), acorde a lo sugerido por los datos previamente analizados.

\section{Técnica de colección de datos}

Los datos fueron recolectados a través de entrevistas en profundidad (Taylor \& Bogdan, 1986), guiadas con una pauta semiestructurada. El guión consultaba por las motivaciones y temores ante la vivencia de la primera maternidad en su contexto de familia simultánea, y los posibles cambios en sus intenciones de tener hijos asociados a este contexto. Se realizaron preguntas sobre el periodo en que comenzaron a pensar en tener un hijo y la secuencia de eventos hasta el momento presente según si estaban decidiendo tener o no un hijo, en proceso de embarazarse o este ya había nacido-, profundizando en sus pensamientos, emociones y acciones, así como en las dinámicas relacionales conyugales y con los hijastros.

\section{Procedimientos de análisis de datos}

Los datos fueron analizados siguiendo rigurosamente los procedimientos de codificación abierta, axial y selectiva definidos por la Grounded Theory (Flick, 2004; Glasser \& Strauss, 1967; Strauss \& Corbin, 1990).

Las transcripciones fueron examinadas línea por línea, codificándose párrafos relevantes, a partir de las preguntas que guiaban el estudio. La codificación abierta fue realizada inicialmente por una de las autoras, y luego dichos códigos fueron analizados en forma conjunta por ambas autoras (triangulación de datos), revisándose los puntos de convergencia y divergencia, estableciéndose la codificación definitiva a la luz de las entrevistas completas y examinándose los sesgos de las autoras. Los conceptos y categorías fueron permanentemente comparados entre sí, para identificar patrones en los datos. Dichos patrones fueron revisados conforme se iban incorporando nuevas entrevistadas.

Avanzada la codificación abierta, se establecieron relaciones entre los datos y se generaron hipótesis, las que se fueron contrastando con la inclusión de nueva información. Por ejemplo, se contrastaron las experiencias de mujeres que habían tenido su hijo y aquellas que aún no lo hacían, determinándose los puntos de encuentro y divergencia entre ellas. Esto dio origen a varios modelos de codificación axial y un esquema selectivo. 


\section{Resultados}

Este articulo muestra la experiencia de un grupo de mujeres respecto de su primera maternidad en familia simultánea. Se detalla el proceso de llegar a ser madre, sus motivaciones y temores, dando especial atención a cómo varían de acuerdo a la presencia de los hijastros y a la cualidad percibida de sus relaciones con ellos.

\section{CuAdro 1}

Motivaciones y temores "universales" para tener un hijo

\section{Motivaciones y temores frente a la primera maternidad en familia simultánea}

Las mujeres identifican diversos motivos para traer un hijo a la vida, entre ellos, la satisfacción de su necesidad de cuidar a otros y de trascender. A nivel relacional, consideran que el nacimiento de un hijo consolida la relación de pareja y da forma a la familia (ver Cuadro 1).

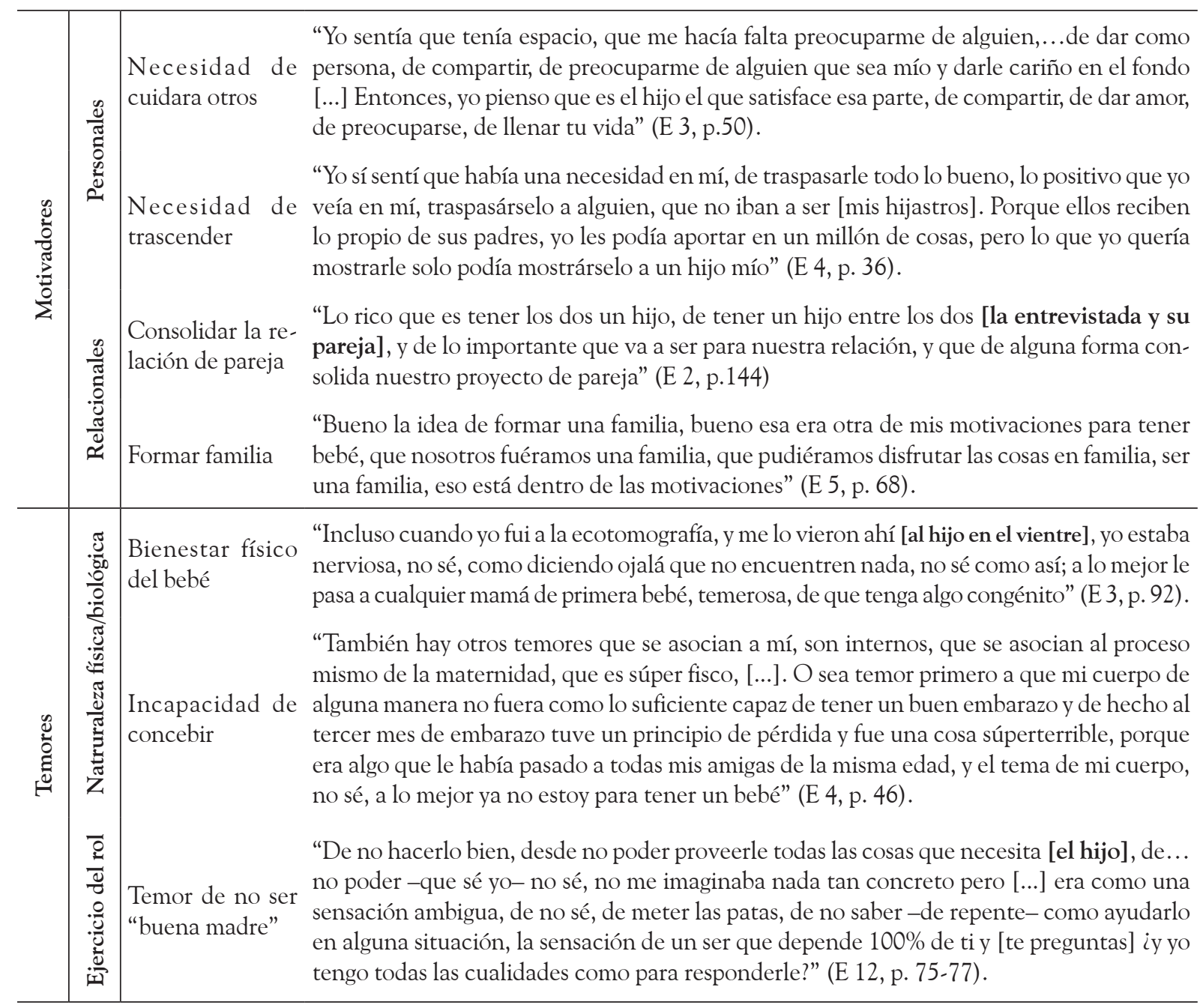

Fuente: elaboración propia. 
Junto a estas motivaciones que pueden presentarse en cualquier tipo de familia, el contexto de familia simultánea adhiere motivaciones específicas para tener hijos (ver Cuadro 2). Así, la motivación de tener un hijo para consolidar la familia adquiere diferentes significados: mi propia familia o una nueva familia.

La motivación de tener un hijo para formar la propia familia, establece una clara diferencia entre el núcleo familiar de la entrevistada y la anterior familia de su pareja. En cambio, la motivación de formar una nueva familia, representa un deseo de integración, construir una familia que abarca a la entrevistada, su pareja y al conjunto de hijos involucrados.

Otras motivaciones para tener un hijo en común al interior de este tipo de familia, se relacionan con la búsqueda de equilibrios entre los adultos implicados en el sistema binuclear. De esta manera, la maternidad es concebida como una forma y oportunidad de equilibrar a las entrevistadas respecto de su pareja, permitiendo que ambos ejerzan roles parentales, es decir, con el nacimiento del hijo las entrevistadas desarrollarían por primera vez roles maternales, que les permitirían empatizar con las sensaciones y/o emociones que vivencia su pareja

CuAdro 2

Motivaciones para tener un hijo asociadas al contexto de familia simultánea

\begin{tabular}{|c|c|c|}
\hline 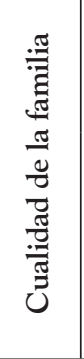 & $\begin{array}{l}\text { Formar la propia fa- } \\
\text { milia }\end{array}$ & $\begin{array}{l}\text { "Yo estaba muy bien, las ganas de estar con él, las ganas de tener un hijo, una familia y de } \\
\text { tener mi familia.... olvidarnos de todo el mundo y hacer nuestra propia casa, nuestra familia } \\
\text { con nuestro hijo" (E 1, p. 137). } \\
\text { "De conformar familia entre nosotros dos y el otro [núcleo familiar]; yo creo que para [los } \\
\text { hijos de mi pareja] también es importante consolidar nuestra relación a través de nuestro } \\
\text { hijo. Que los niños también vean que la separación de sus padres, entre muchas otras cosas } \\
\text { malas, ha traído cosas buenas, una de las cosas buenas es [mi hijo] y -de hecho- ellos están } \\
\text { fascinados por [mi hijo] y él se muere por sus hermanos" (E 2, p. 67). }\end{array}$ \\
\hline 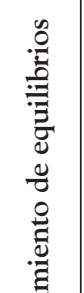 & $\begin{array}{l}\text { Equilibrar a las mu- } \\
\text { jeres con sus parejas } \\
\text { en roles parentales }\end{array}$ & $\begin{array}{l}\text { "Para mí era importante lograr un cierto equilibrio, del punto de vista de que él ya tenía hijos } \\
{[\ldots . .] \text {, yo creo que para mí y para nosotros también -lo hemos discutido así- que es importante }} \\
\text { lograr un equilibrio también, de alguna forma nos equilibraba la posición de ambos, el que } \\
\text { tuviéramos hijos juntos [...] -te digo- pero más fácil hubiera sido que yo también hubiera } \\
\text { tenido hijos de un matrimonio anterior, porque -en el fondo- es mucho más fácil ponerte en } \\
\text { el zapato del otro, porque para mí es bastante desconocido, si yo no tengo hijos, ver, sentir } \\
\text { que es lo que [mi pareja] siente frente a estos niños" (E 2, p. 13). }\end{array}$ \\
\hline 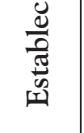 & $\begin{array}{l}\text { Equilibrar a las mu- } \\
\text { jeres con las madres } \\
\text { de sus hijastros/as }\end{array}$ & $\begin{array}{l}\text { "Ahora uno tiene también un hijo con esa pareja, en el fondo no solo es la expareja quien } \\
\text { tiene el vínculo del hijo, y uno tiene el vínculo del amor no más; pero cuando hay un hijo } \\
\text { establece un vínculo más fuerte, yo también tengo el vínculo del hijo" (E 1, p. 169). }\end{array}$ \\
\hline 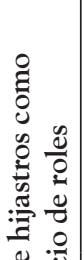 & $\begin{array}{l}\text { Desarrollo del pro- } \\
\text { pio rol materno }\end{array}$ & $\begin{array}{l}\text { "En el momento en que empezamos a vivir juntos, [los hijos de mi pareja] se transformaron } \\
\text { en una parte medular de nuestra cotidianeidad. Yo los conocí superchiquititos, y de alguna } \\
\text { manera empezó como a formarse un rol maternal, que yo no lo buscaba porque todavía no } \\
\text { era tema en nuestra familia, pero claro estando ellos, yo tenía una imagen maternal que } \\
\text { tenía que -de alguna manera- empezar como a desarrollarla, y yo creo que ese fue como un } \\
\text { factor superimportante" (E 4, p.24). }\end{array}$ \\
\hline 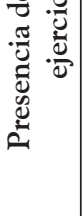 & $\begin{array}{l}\text { Posibilidad de que } \\
\text { la pareja ejerza co- } \\
\text { tidianamente el rol } \\
\text { paterno }\end{array}$ & $\begin{array}{l}\text { "Piensa tú que él, yo me pongo a pensar en él, dejó de ver a su hijo a los } 3 \text { años, [es distinto] } \\
\text { tener a tu hijo viviendo todos los días con él, y eso me da felicidad, porque ahora [que na- } \\
\text { ció el hijo en común] lo veo contento, comparten y correr, porque eso no lo vivió también, } \\
\text { desde ese punto de vista de satisfacer una necesidad de mi marido, que yo quiero que esté } \\
\text { contento" (E 3, p. 70). }\end{array}$ \\
\hline
\end{tabular}

Fuente: elaboración propia. 
en relación con sus hijos. En segundo lugar, como una forma de equilibrar a las entrevistadas ante la madre de los hijos de su pareja, ya que este primer hijo en común establecería un vínculo madre-hijo en cada uno de los núcleos familiares del sistema binuclear.

La existencia de ciclos de presencia-ausencia de los hijastros, quienes visitan periódicamente el núcleo familiar de las entrevistadas, actúa motivando la propia maternidad en dos formas diferentes. En primera instancia, vivir en lo cotidiano con los hijos de la pareja (aunque sea sólo algunos fines de semanas o en períodos de vacaciones) actúa desarrollando en las entrevistadas el de rol cuidado hacia otros, lo que desde sus percepciones las acerca a su propia maternidad. En forma diferente, el percibir que su pareja no puede disfrutar de su hijo cada día, genera en una de las entrevistadas el deseo de dar a la pareja la posibilidad de vivir en lo cotidiano junto a un hijo.

Junto a estas motivaciones para la maternidad, coexisten temores de diversa índole, que no difieren de los temores que cualquier mujer pudiera tener frente a la maternidad (ver Cuadro 1, Temores). Los temores más comunes giran en torno a la incapacidad de concebir el hijo deseado y llevar a buen término el embarazo, así como la preocupación por el bienestar físico del bebé por nacer. A ello se suma el temor de no ser "buenas madres", es decir, no poder cumplir efectivamente con lo que significa hacerse cargo del bebé.

Ahora bien, en función de su contexto familiar específico, aparece el temor de que sus parejas no vivan con la misma intensidad esta experiencia tan importante para las entrevistadas, ni con la misma urgencia que estas tienen, pues ya han vivido previamente la experiencia de ser padres (ver Cuadro 3).

Esta misma experiencia previa de sus parejas, es lo que las hace temer que la crianza del hijo en común se transforme en una negociación permanente entre la experiencia del padre y la inexperiencia de madre primeriza, a partir de lo cual el primero pudiera ejercer un rol preeminente en la crianza. En forma opuesta, las entrevistadas temen que ellos asuman solo un rol secundario, dado que ya se han acostumbrado como padres separados, a cuidar y responsabilizase por sus hijos en general solo algunos fines de semana, y no asuman el cuidado del nuevo hijo en el día a día, dejándoles a ellas la mayor responsabilidad de la crianza.

Otros miedos adicionales fueron nombrados por las entrevistadas, los cuales serán descritos luego, enfatizando si estos varían de acuerdo a la cualidad atribuida a las relaciones entre las entrevistadas y sus hijastros.

Por último, es necesario señalar que las mujeres entrevistadas mencionaron las motivaciones y temores antes especificados, en forma independiente de si ellas habían sido madres o no al momento de la entrevista. Sólo se presentó una excepción: la motivación por encontrar un balance entre los adultos presente en el sistema binuclear fue mencionada solamente por mujeres que ya habían sido madres al momento de la entrevista.

\section{Relación entre la presencia de los hijastros y el proceso de llegar a "ser madre"}

En general, las entrevistadas perciben que la presencia de los hijos de su pareja no afecta su decisión de tener su primer hijo. Tal como lo dice una de ellas: "Ellos no afectan, o sea el que yo quiera o pueda, ellos no afectan en nada. Ni en mi pareja, ni en mí misma" (E 15, p. 132). En cambio, las entrevistadas sí indican que su presencia afecta directamente la cantidad total de hijos que ellas tendrán, disminuyendo su número, dadas las exigencias económicas y de tiempo que sus parejas tienen con sus hijos de relaciones previas. Un ejemplo de ello aparece en el siguiente relato: "Existe la posibilidad de que no tengamos más hijos [...], además porque cada hijo en este país, sale muy caro, y él ya tiene 3 , entonces [él] me dice: claro para ti es el segundo, pero para mí van a ser cuatro" (E 7, p. 64).

$\mathrm{Al}$ analizar la vivencia de las entrevistadas en el proceso de llegar a ser madres, a la luz de la cualidad percibida de la relación con sus hijastros, surge un aspecto en común y se delinean dos contrapuntos relevantes: el primero, relacionado con los temores ante la maternidad, el segundo, respecto de 


\section{CuAdro 3}

Temores específicos ante la primera maternidad en el contexto de familia simultánea

\begin{tabular}{c|l|l}
\hline & "Y de alguna forma tienes el temor -y de hecho yo creo que ocurre- que tu pareja ya ha \\
tenido otros hijos antes, no vive con la misma intensidad todo esto, porque me imagino \\
que tu también a tu tercer hijo ya no lo vivirás de la misma manera, entonces eso es un \\
miedo asociado, es una situación compleja" (E 2, p. 23).
\end{tabular}

Fuente: elaboración propia.

la forma de comunicar la intención de ser madre (ver Cuadro 4),

Las mujeres que relatan tanto buenas como malas relaciones con sus hijastros, comparten el temor de que sus hijos no sean queridos o aceptados por estos.

Las mujeres que relataban relaciones positivas con los hijos de sus parejas, es decir, relatan sentir afecto por sus hijastros y manifiestan disfrutar las actividades conjuntas, presentan temores centrados en el bienestar del conjunto de los miembros de la familia, específicamente:

1. Temor de que los hijos de la pareja se sientan excluidos con la llegada del nuevo miembro y/o preocupación por sobre cuál será el impacto en ellos.

2. Temor de cómo ellas mismas se equilibrarán en el ejercicio de sus roles luego del nacimiento del hijo, dando a cada niño el espacio que le corresponde, o cómo sus parejas lograrán este equilibrio.

En cambio, las mujeres que planteaban tener malas relaciones con los hijos de sus parejas, relatando que tienen conflictos abiertos con ellos y/o que sienten su rechazo, los temores están centrados, mayormente, en el bienestar propio y de su progenie:

1. Se impone el temor a que los hijos de la pareja sean mala influencia para sus propios hijos. Este temor expresa no sólo desacuerdo con la forma en que estos hijos son criados, sino que deja entrever una sensación de amenaza hacia los propios valores que ellas desean transmitir a sus hijos y, en última instancia, hacia sus propias creencias y valores. 


\section{CUADRO 4}

Relaciones entre la presencia de los hijastros y el proceso de "llegar a ser madre"

\begin{tabular}{|c|c|c|}
\hline & Buenas Relaciones Mujeres/hijastros & Malas Relaciones Mujeres/hijastros \\
\hline \multicolumn{3}{|c|}{ Aspectos Comunes } \\
\hline $\begin{array}{l}\text { Temor respecto } \\
\text { de los hijastros }\end{array}$ & $\begin{array}{l}\text { "Yo creo que me costó muchísimo darme cuenta qu } \\
\text { [...] y nació, y fue difícil, igual fue difícil, pero yo ig } \\
\text { no la sintieran como una hermana también" (E } 4, \mathrm{p} \\
\text { "Que [mi hijastra] no la aceptara; al principio fue } \\
\text { familia feliz, o sea que [mi hijastra] viniera a la casa, } \\
\text { tirio: 'me toca ir a la casa de mi papá, y está esta ot } \\
\text { (E 13, 44-66). }\end{array}$ & $\begin{array}{l}\text { gual sentía el temor del rechazo de partida, de que } \\
\text { p. 58). } \\
\text { terrible }[\ldots . . .] \text { porque para mí lo ideal es tener una } \\
\text { a, que le gustase venir para acá, que no sea un mar- } \\
\text { tra niña acá que me está quitando mi, mi papá'..." }\end{array}$ \\
\hline \multicolumn{3}{|c|}{ Contrapunto } \\
\hline $\begin{array}{l}\text { Temor respecto } \\
\text { de los hijastros }\end{array}$ & $\begin{array}{l}\text { "Yo creo que hemos tenido harto cuidado y [mi } \\
\text { pareja] también le ha preocupado, que los niños } \\
\text { no se sientan como dejados de lado porque aparece } \\
\text { un nuevo hijo con el cual él comparte todos los } \\
\text { días" (E 2, p. 32). }\end{array}$ & $\begin{array}{l}\text { "Que no sea una buena influencia [para mi hijo], } \\
\text { porque claro, mi idea es que los hermanos grandes } \\
\text { son como ídolos, entonces si yo no estoy de acuerdo } \\
\text { con la educación de [mi hijastro], para mí es una es- } \\
\text { pecie de ídolo negativo entre comillas" (E 5, p. 46). }\end{array}$ \\
\hline $\begin{array}{l}\text { Temor respecto } \\
\text { del logro de } \\
\text { equilibrios de } \\
\text { los miembros. }\end{array}$ & $\begin{array}{l}\text { “...era cuánto tiempo le dedicará a cada una, por- } \\
\text { que [mi pareja] es bien apegado a la otra hija, no } \\
\text { sé ahora un poco menos, se han visto menos, pero } \\
\text { siguen siendo súper [cercanos], entonces ahí yo } \\
\text { tenía el temor de si iba a ser igual con la mía que } \\
\text { con la primera de él” (E 1, p. 89). } \\
\text { "entonces yo creo que una presencia de un niño } \\
\text { [hijo en común], que está todo el tiempo con noso- } \\
\text { tros va a ser muy duro para [mi hijastro], y también } \\
\text { va a ser un desafío para mi, para darle a él el lugar } \\
\text { que le corresponde, y a mi hijo también, va a ser } \\
\text { difícil...”(E 10, p. 42). }\end{array}$ & $\begin{array}{l}\text { "Mi fantasía catastrófica de lo que puede pasar [a } \\
\text { futuro] es que [mi hijastra] se embarace y su padre } \\
\text { [mi pareja] va a cargar con esa guagua también y } \\
\text { con los gastos y con mantener a esta mamá con su } \\
\text { hija, con su nieta... entonces como desde ahí yo } \\
\text { siento que yo voy a cargar muy sola con el hijo que } \\
\text { nosotros podríamos tener [...] contactarme con } \\
\text { todo esto otro, con lo pedestre, de las platas, de } \\
\text { verme a mi misma peleando entre comillas por los } \\
\text { pesos, que finalmente no son los pesos en realidad, } \\
\text { sino los espacios, las prioridades, a mí me deprime" } \\
\text { (E } 11, \text { p. 56-74). }\end{array}$ \\
\hline $\begin{array}{l}\text { Radicalización } \\
\text { de las malas } \\
\text { relaciones }\end{array}$ & & $\begin{array}{l}\text { "[Pienso que mis hijastros] no me van a querer ver } \\
\text { pero ni en pintura, yo voy a ser la mala de la pelí- } \\
\text { cula, yo creo que no me van a querer ver de hecho, } \\
\text { casi como que no van a ir los fines de semana, van } \\
\text { a empezar a buscar excusas para no ir los fines de } \\
\text { semana a mi casa" (E 14, p. 78). }\end{array}$ \\
\hline
\end{tabular}

Fuente: elaboración propia.

2. Temor a que sus parejas no logren equilibrarse ante las múltiples exigencias y responsabilidades -económicas y distribución de tiempoque implica conformar un sistema binuclear con hijos en ambos grupos familiares, perjudicando a su núcleo familiar.

3. Temor a profundizar las malas relaciones existentes entre ellas y los hijos de su pareja. Temor que se basa en las experiencias que viven en la interacción con sus hijastros, y que se proyecta en el nuevo escenario a desarrollarse, atentando directamente contra su bienestar emocional.

El segundo contrapunto surge al momento de la comunicación de la intención de ser madres y la llegada del bebé. Las mujeres que refieren relaciones 
positivas con los hijos de sus parejas, socializan la noticia considerando cuál será el mejor momento para dárselas a conocer, pensando en la edad de los niños o en las situaciones particulares que están viviendo (por ejemplo, la llegada de un hermano por parte de la madre). En cambio, las entrevistadas que describen presentar malas relaciones con ellos, prefieren que sea la pareja quien comunique el hecho, y dilatan lo más posible la comunicación para evitar más conflictos entre ellos.

\section{El proceso y las dificultades para llegar a ser madre en el contexto de familia simultánea}

El proceso de llegar a ser madre aparece como invisible en la medida que la experiencia de las mujeres no presente dificultades, pero frente a ellas es posible diferenciar hitos secuenciales. Este proceso es válido en la medida que el llegar a tener hijos es una acción planificada, lo que aconteció en la mayoría de las entrevistadas.
Como todo proceso, la secuencia estipulada indica los pasos a ser recorridos, sin embargo, se puede avanzar de hito en hito, en la medida que se van cumpliendo, o retroceder en ellos. Esto último va aparejado de sentimientos negativos en las mujeres, como pena, enojo o decepción.

La maternidad se instala sobre la existencia de una relación positiva de pareja, en la cual debe existir una confluencia en el deseo de ser padres. El hecho de compartir el deseo de ser padres puede ser asumido como preexistente, o explicitado por uno o ambos miembros de la relación de pareja, generalmente temprano en el curso de la relación. Tal como lo plantea una de las entrevistadas: "Es como curioso, porque yo creo que lo conversamos, tengo la sensación de haberlo conversado mucho, pero ahora que tú me haces la pregunta, creo que no lo conversamos tanto, y creo que coincidimos en un deseo que era común" (E 4, p. 83).

No obstante, la presencia de conflictos entre los hijastros y las entrevistadas puede volver inestable

Figura 1

El proceso y las dificultades para llegar a ser madre en el contexto de familia simultánea

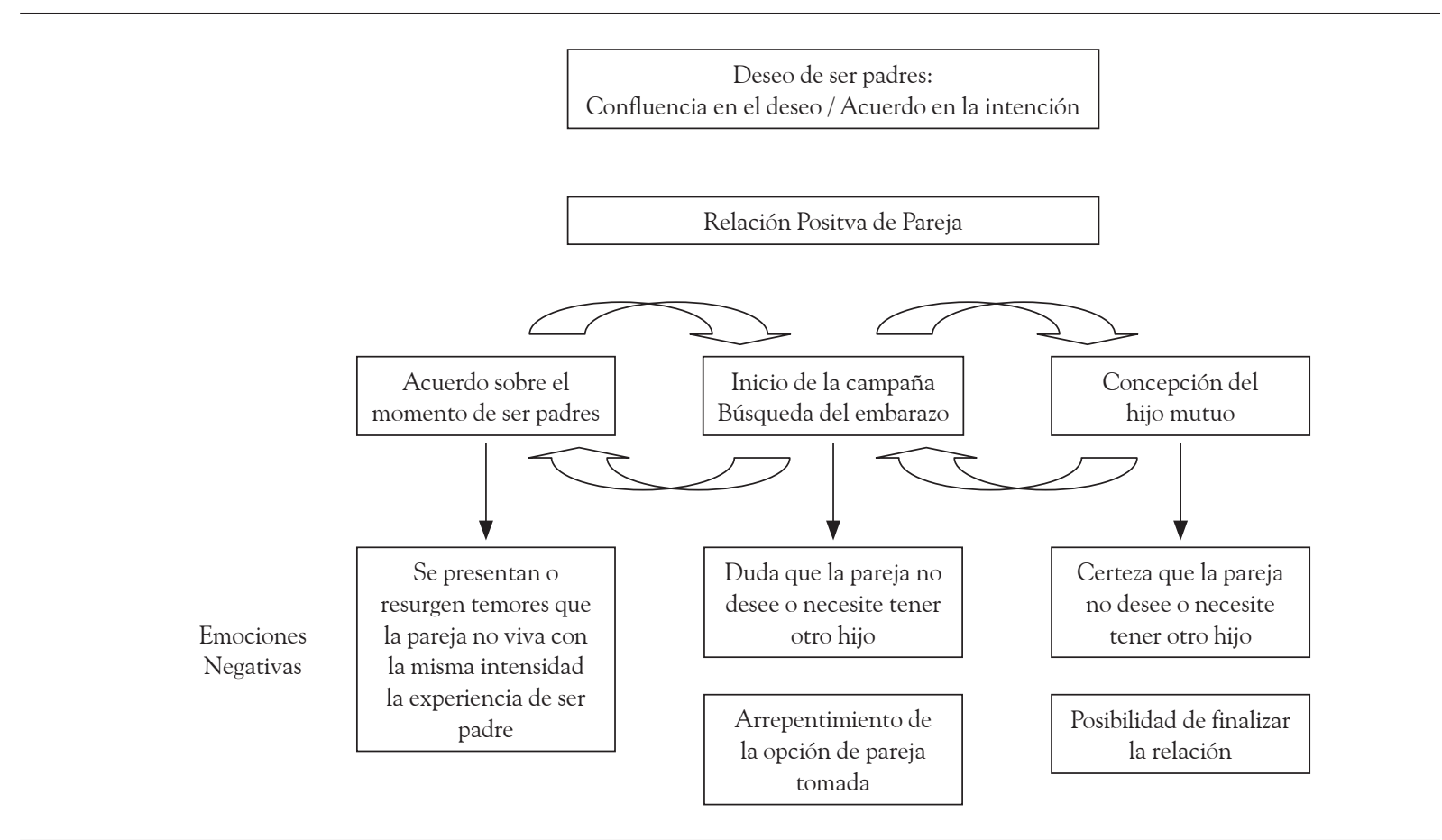

Fuente: elaboración propia. 
la relación de pareja, haciendo desaparecer una de las condiciones sindicada como necesaria para el inicio del proceso de tener hijos.

El deseo de ser madre se refiere específicamente a la expresión de un deseo o intención por parte de las entrevistadas, que para traducirse finalmente en la llegada de un bebé, necesita como primer paso, determinar cuál será el momento en que serán madres, para con ello dar lugar a la "campaña para tener hijos" que finaliza con el logro de la concepción y nacimiento.

El primer desafío es establecer un acuerdo sobre el momento de ser padres, instalándose la pregunta itenemos hijos ahora o más adelante? Determinar cuál es el momento más adecuado para tener hijos es reportado por las entrevistadas como un hito difuso. Les es difícil identificar y/o recordar un momento exacto, más bien tienen la impresión que es la confluencia de una serie de aspectos que favorecen y gatillan el inicio de la campaña, o que la demoran. Esta difusión se aprecia claramente en el siguiente comentario de una de las entrevistadas:

Entonces, yo creo, sabes que no sé exactamente en qué lo decidimos. Ahora que lo pienso, de alguna forma lo había estado posponiendo un poco, pero estaba ahí el tema [de tener un bebé], en el fondo, decidimos... es que no me acuerdo cómo fue, pero hay algo como que hubo un proceso, pero claramente la cosa ambiental [amigos y familiares teniendo hijos] a mí me hizo pensar más [en decidir tener un bebé] (E 16, p. 94).

Mayor premura para concretar la maternidad aparece cuando las entrevistadas ven a sus amigos y familiares directos teniendo y disfrutando de sus hijos; cuando desean tener más de un hijo, y si, en función de su edad, piensan que tendrán mayores dificultades para concebir y llevar a buen término dicho embarazo.

La presencia de los hijastros opera demorando la concreción de la maternidad, al intervenir en la consolidación de la pareja o familia. Por ejemplo, una de las entrevistadas expresó que primero es necesario lograr un funcionamiento familiar que les permita afrontar las exigencias de los hijastros, quienes poseen dos núcleos familiares, antes de tener un propio hijo. En las palabras de una entrevistada:

Yo creo que en un inicio lo conversamos, que no era urgente tener hijos luego y -de hecho- también acordamos que nos gustaría un tiempo estar solos, por una necesidad de consolidarnos como pareja, y -además- consolidar este nuevo sistema, de ver cómo funcionábamos, porque es complejo funcionar con niños que van y vienen (E 2, p. 48).

Otra entrevistada señala que la presencia de los hijastros afecta la consolidación de la pareja al contar con menos tiempo para estar ellos a solas:

O sea si fuera por [mi marido] yo me hubiera embarazado el día de la luna de miel, en cambio yo sentía que teníamos que tener un tiempo solos como pareja. A lo mejor la presencia de [mi hijastro] influyó en que yo quisiera esperar un poco más para tener un bebé, porque yo sentía la necesidad de estar un tiempo solos, estamos solos un fin de semana por medio, entonces eso me hizo querer aplazar, yo sentía esta necesidad de conformarnos primero como pareja, acostumbrarnos a vivir juntos, si a lo mejor él [hijastro] no hubiera estado, yo habría esperado menos tiempo quizás para tener un bebé (E 5, p. 54).

Las entrevistadas señalan dos trayectorias al momento de lograr acuerdo sobre el momento propicio para tener hijos. El acuerdo inmediato "ahora es el momento" para tener este hijo, da inicio a la campaña. En cambio, cuando se determina esperar un tiempo y ser padres más adelante, se hacen necesarios establecer nuevos acuerdos para comenzar posteriormente la campaña para tener hijos.

El inicio de la campaña para tener hijos incluye el manejo de los aspectos biológicos necesarios para el logro de la concepción. La mayoría de las entrevistadas señala la visita al médico como el hito inicial o al menos importante dentro de la campaña; lo cual se acompaña de descontinuar el uso de métodos anticonceptivos.

La capacidad reproductiva - entendida como la capacidad del organismo de concebir y llevar 
a buen término un embarazo - tanto real como percibida, determina la velocidad con que se pasa de una etapa a otra. En la medida que las entrevistadas perciben mayores dificultades en el logro de la concepción, buscan el acuerdo de tener hijos temprano en la relación, disponiendo así de más tiempo para lograr el embarazo. En la medida que no se duda de esta capacidad reproductiva, no presentan esta premura para embarazarse.

Si no se logra la concepción, comienzan a aparecer en las entrevistadas dudas sobre si la pareja desea tener otro hijo, y les surgen ideas relativas a que hubiese sido mejor establecer una relación de pareja con un hombre que no tuviera hijos. Estas ideas desaparecen en la medida que se logra la concepción y nacimiento del hijo, pero se radicalizan en la medida que esto no acontece. Incluso, aparece la certeza de que la pareja no desea tener otro hijo y surge la posibilidad, en al menos una de las entrevistadas, de finalizar la relación.

\section{Discusión}

Los resultados muestran que tener su primer hijo implica para las entrevistadas una serie de hitos secuenciales que conforman un proceso. Este proceso se inicia con la búsqueda de un acuerdo sobre el momento para ser padres, continúa con el inicio de la "campaña para tener hijos" y finaliza con la concepción, y posterior nacimiento, del primer hijo en común; tiene a su base la existencia de una relación positiva de pareja y la confluencia y/o acuerdo en el deseo de tener hijos. Pero la vivencia de este proceso por parte de las entrevistadas, y su curso, variarían en función de la cualidad percibida de las relaciones con los hijastros.

$\mathrm{Al}$ igual que en el caso de las familias nucleares, la dificultad para concebir trae aparejada emociones negativas, conflictos conyugales y la posibilidad de finalizar la relación (Domínguez, 1995). Adicionalmente, y ligado al contexto de familia simultánea, en el imaginario de las mujeres estudiadas surgen ideas que cuestionan la relevancia, el deseo y la necesidad de sus parejas de tener hijos, ideas que al presentarse profundizan el malestar y los conflictos que caracterizarían el tratar de ser padres cuando hay problemas de fertilidad.

Tal como lo plantea Miller (1992), cuando llega el minuto y bajo condiciones adecuadas, las intenciones de tener hijos se transforman a conducta proceptiva. En el caso particular de las mujeres entrevistadas, la presencia de sus hijastros tendría impacto en la determinación del momento adecuado para tener hijos, demorándolo en general. Además, cuando ellas consideran que tienen malas relaciones con los hijos de sus parejas, se ve interferida su relación de pareja, que tal como se mencionó anteriormente, es la base o la condición que soporta todo el proceso de maternidad en este contexto de familia simultánea. De hecho, los conflictos entre padrastros/hijastros son una de las principales dificultades al interior de las familias simultáneas, siendo uno de sus mayores desafíos el lograr relaciones satisfactorias entre ellos (Visher \& Visher, 1988).

En las mujeres estudiadas, las intenciones de tener hijos son altas, concordando con lo planteado por Stewart (2002) y Thompson y Allen Li (2002), quienes señalan que los hijastros tienen un bajo impacto sobre las intenciones de tener hijos, en el caso particular de las familias simultáneas que no tienen un "hijo en común". No obstante, su concreción implica superar las diferentes dificultades - ya sea biológicas o relacionales - que surgen durante el proceso de llegar a ser madres. La presencia de los hijastros, afectaría entonces reduciendo la probabilidad de ocurrencia de un segundo embarazo, dado los costos asociados a la criaza de una progenie de varios hijos.

Particular relevancia adquiere la edad cronológica de las mujeres que forman este tipo de familia. Cuando estas son mayores de 30 años, sienten una premura biológica para concretar el embarazo - tal como lo reportaron las entrevistadas del presente estudio - entrando en conflicto con la necesidad relacional de consolidar el funcionamiento familiar, lo cual requeriría más tiempo en las familias simultáneas. Así, por una parte vivencian un "último plazo biológico”, que establece límites objetivos y presiones subjetivas para la concreción de la 
concepción y embarazo, y, a la par, necesitan una mayor cantidad de tiempo para consolidar su familia, ya sea por contar con menos tiempo solos al recibir sistemáticamente a los hijos de la pareja, o al requerir de tiempo para lograr un funcionamiento adecuado entre todos.

Más relevante en la vivencia y curso de este proceso, que la mera presencia de los hijos de la pareja, es la cualidad de las relaciones entre las entrevistadas y sus hijastros. La percepción de malas relaciones entre ellos hace que el proceso de ser madres se acompañe con temores de que las entrevistadas, sus hijos y/o su núcleo familiar sean dañados, generando reacciones de autodefensa. En cambio, cuando estas relaciones son percibidas como buenas, el proceso de tener un hijo biológico va acompañado por temores sobre la búsqueda de un nuevo equilibrio entre el conjunto de los miembros del sistema, que las incluye a ellas, sus parejas, los hijastros y el "hijo en común".

Las mujeres que reaccionan defensivamente no encontrarían en sus parejas un referente que valide sus puntos de vista, ya que hacerlo los pone en un dilema: si validan la experiencia de sus mujeres actuales entran en conflicto con sus hijos, y si no lo hacen, se transforman en hombres poco comprensivos, lo que refuerza los temores de sus mujeres, generando una actitud aún más defensiva.

El afrontar las tensiones propias del embarazo y parto, y superar aquellas adicionales que conllevan las malas relaciones entre las entrevistadas y sus hijastros, exige a la pareja conyugal, requiriendo que estas sean capaces de manejar conflictos y afrontar constructivamente sus dificultades.

En el marco del objetivo principal de este artículo, que era dar cuenta de la vivencia del proceso de ser madre en un contexto particular de familia simultánea, es que la elección muestral consideró mujeres que estuvieran viviendo este proceso y aquellas que ya lo hubieran experimentado, buscándose información heterogénea que permitiera ver un desarrollo temporal y posibles trayectorias diversas en las familias. Desde esta perspectiva, se intencionó el muestreo hacia mujeres que percibieran sus relaciones familiares con y sin dificultades.
Sin embargo, particularmente las mujeres que percibían tener dificultades relacionales con los hijos de sus parejas, son pocas en este estudio, por lo que resultaría sumamente interesante profundizar en esta línea en investigaciones futuras.

El carácter retrospectivo de algunas de las entrevistas (en el caso de aquellas mujeres que ya habían tenido sus hijos al momento de las entrevistas), podría considerarse una limitación, ya que los recuerdos de la época en que estaban decidiendo e intentando tener su primer hijo no fueran totalmente exactos o precisos. No obstante, sus aportes orientaron la generación de preguntas específicas sobre el proceso de maternidad y fueron consistentes con la información recolectada de mujeres que estaban actualmente viviendo este proceso.

La saturación de la información lograda a lo largo del estudio, permite concluir que en este tipo de familias simultáneas se adhieren motivaciones y temores particulares asociados a la primera maternidad, y que la presencia de los hijastros tiene influencia en algunos aspectos del proceso de llegar a ser madre. Adicionalmente, se puede concluir que la cualidad de las relaciones entre las mujeres y sus hijastros/as operaría "cualificando" la experiencia, mostrando que afecta la manera en como ellas interpretan los eventos que acontecen en los tópicos relevantes en su primera maternidad.

Sin embargo, se sugiere que investigaciones futuras profundicen en estos hallazgos incluyendo la perspectiva de otros miembros de la familia, ya que la visión de las parejas e hijastros/as puede complementar o poner en discusión algunos de los elementos señalados en el presente artículo.

No obstante, las limitaciones y desafíos de investigación señalados, los hallazgos permiten establecer líneas orientadoras en el ámbito de la salud mental. Los resultados indican que el tener el "primer hijo en común" a ambos miembros de la pareja, puede ser una experiencia positiva y de crecimiento, pero también puede transformarse en fuente de malestar para las mujeres y, adicionalmente, afectar el funcionamiento conyugal. Particularmente en el caso de las mujeres, conocer que se presentan temores particulares ligados al con- 
texto familiar, y que emociones como la decepción o desesperanza son vividas por otras mujeres en situaciones similares a las suyas, les permite "normalizar" su experiencia. En este mismo sentido, los resultados obtenidos constituyen aportes para la práctica clínica, ya que a partir de las experiencias de las mujeres entrevistadas, surgen tópicos particulares que deben considerarse al momento de realizar intervenciones, ya sea con las mujeres o en una intervención familiar. Por último, los resultados deben darse a conocer al personal de salud que se relaciona con mujeres que tienen hijos en el contexto de familia simultánea, permitiéndoles poner atención y/o identificar fuentes adicionales de estrés durante el embarazo y parto.

\section{Referencias}

Ambert, A. (1986). Being a stepparent: Live-in and visiting children. Journal of Marriage and the Family, 48, 795-804.

Bumpass, L. L., Kelly, R. \& Sweet, J. A. (1995). The changing character of stepfamilies: Implications of cohabitation and nonmarital childbearing. Demography, 32, 425-436.

Coleman, M., Ganong, L. \& Fine, M. (2000). Reinvestigating remarriage: Another decade of progress. Journal of Marriage and the Family, 62, 1288 -1307.

Cowan, C. P. \& Cowan, P. A. (1995). Interventions to ease the transition to parenthood: Why they are needed and what they can do. Journal of Marriage and the Family, 44, 412-423.

Crosbie-Burnett, M. (1989). Application of family stress theory to remarriage: A model for assessing and helping stepfamilies. Family Relations, 38, 323-331.

Chile, Instituto Nacional de Estadísticas. (2005). Anuario de Estadísticas Vitales: 2003. Santiago de Chile: Autor.

Chile, Servicio Nacional de la Mujer. (2002). Hombres y mujeres: como ven hoy su rol en la sociedad y en la familia. Santiago: Autor.

Demo, D. H. \& Cox, M. J. (2000). Families with young children: A review of research in the 1990s. Journal of Marriage and the Family, 62, 876-895.
Dietz-Omar, M. (1991). Couple adaptation in stepfamilies and traditional nuclear families during pregnancy. Nursing Practice, 4, 6-10.

Domínguez, R. (1995). Cuando es difícil ser familia. Aspectos psicosociales de la infertilidad. Proposiciones, 26, 235-242.

Downs, K. (2003). Family commitment, role perceptions, social support, and mutual children in remarriage: A test of uncertainty reduction theory. Journal of Divorce and Remarriage, 40, 35-53.

Duberman, L. (1973). Step-kin relationship. Journal of Marriage and the Family, 35, 283-292.

Fine, M. (1995). The clarity and content of the stepparent role: A review of literature. Journal of Divorce and Remarriage, 24, 19-34.

Flick, U. (2002). An introduction to qualitative research. London: Sage.

Ganong, L. \& Coleman, M. (1988). Do mutual children cement bonds in stepfamilies? Journal of Marriage and the Family, 50, 687-698.

Glasser, B. \& Strauss, A. (1967). The discovery of Grounded Theory. Chicago: Aldine.

Haurin, R. J. (1994, mayo). Determinants of Fertility in Remarriage: Outcomes of White American Experience. Ponencia presentada en el Annual Meeting of Population Association of America, Miami, FL, USA.

Loomis, L. S. \& Landale, N. S. (1994). Nonmarital cohabitation and childbearing among black and white American women. Journal of Marriage and the Family, 56, 949-962.

Miller, W. (1992). Personality traits and developmental experiences as antecedents of childbearing motivation. Demography, 29 (2), 265-285.

Muñoz, M. \& Reyes, V. (1997). Formas de separación matrimonial y sus principales consecuencias en Chile. Revista de Trabajo Social, 69, 69-89.

Olson, D. H., Russell, C. S. \& Sprenkle, D. H. (1983). Circumplex model of marital and family systems: VI. Theoretical update. Family Process, 22, 69-83.

Rogers, S. \& White, L. (1998). Satisfaction with patenting: the role of marital happiness, family structure, and parents' gender. Journal of Marriage and the Family, 60, 293-308. 
Stewart, S. (2002). The effect of stepchildren on childbearing intentions and births. Demography, 39, 181-197.

Stewart, S. (2005). How the birth of a child affects involvement with stepchildren. Journal of Marriage and Family, 67, 461-473.

Strauss, A. \& Corbin, J. (1990). Basic of qualitative research: Grounded Theory procedures and techniques. Newbury Park, CA: Sage.

Taylor, S. \& Bogdan, R. (1984). Introduction to qualitative research methods. The search for meanings. New York: John Wiley \& Sons.

Tironi, E., Gubbins, V., Larrañaga O., Teitelboim, B., Bravo, D. \& Valenzuela, E. (2003). Cuánto y cómo cambiamos los chilenos balance de una década: censos
1992-2002. Santiago: Comisión BicentenarioINE.

Thompson, E. \& Allen Li, J. C. (2002). Her, his, and their children: Childbearing intentions and births in stepfamilies. NSFH Working Paper 89. Recuperado el 20 de febrero de 2008, de http://www.ssc.wisc. edu/cde/nsfhwp/nsfh89.pdf

Wineberg, H. (1990). Childbearing after remarriage. Journal de Marriage and the Family, 52, 31-38.

Wineberg, H. (1992). Childbearing and dissolution of the second marriage. Journal of Marriage and the Family, 54, 879-887.

Visher, E. \& Visher, J. (1988). Old loyalties, new ties. Therapeutic strategies with stepfamilies. New York: Brunner-Mazel. 